INPLASY

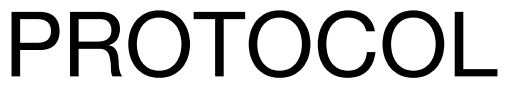

To cite: Yi et al. Efficacy and safety of surgical ablation concomitant to coronary artery bypass grafting for atrial fibrillation: a meta-analysis. Inplasy protocol 202140081. doi:

10.37766/inplasy2021.4.0081

Received: 15 April 2021

Published: 16 April 2021

Corresponding author:

Tao You

youtao2016@126.com

Author Affiliation:

Department of Cardiovascular

Surgery, Gansu Provincial

Hospital

Support: GSWSKY2016-04.

Review Stage at time of this submission: Formal screening of search results against eligibility criteria.

Conflicts of interest:

None declared.

\section{Efficacy and safety of surgical ablation concomitant to coronary artery bypass grafting for atrial fibrillation: a meta-analysis}

Yi, K¹ Xu, XM²; Xu, JG3; He, SE4; Wang, WX5; Ma, YH6; Gao, J7; Wang, W8; You T9.

Review question / Objective: P: Patients with preoperative atrial fibrillation (AF) and indication for coronary artery bypass grafting (CABG). I: Surgical ablation (SA) concomitant to isolated CABG procedure. $C$ : isolated CABG procedure. $O$ : Freedom from AF (defined as no AF recurrence during followup duration); early mortality (defined as any death within 30 days of CABG or during the CABG admission); long-term mortality (defined as all-cause mortality during follow-up duration); perioperative relevant events (such as renal impairment, permanent pacemaker implantation, stroke or transient ischemic attack, bleeding, sternal wound infection). S: Randomized controlled trial (RCT) or cohort study. Objective: The aim of this systematic review and metaanalysis is to evaluate the efficacy and safety of SA at the time of isolated CABG for preoperative AF patients based on the published literature, and provide reference for clinical decision.

INPLASY registration number: This protocol was registered with the International Platform of Registered Systematic Review and Meta-Analysis Protocols (INPLASY) on 16 April 2021 and was last updated on 16 April 2021 (registration number INPLASY202140081).

\section{INTRODUCTION}

Review question / Objective: P: Patients with preoperative atrial fibrillation (AF) and indication for coronary artery bypass grafting (CABG). I: Surgical ablation (SA) concomitant to isolated CABG procedure. $C$ : isolated CABG procedure. O: Freedom from AF (defined as no AF recurrence during follow-up duration); early mortality 
(defined as any death within 30 days of CABG or during the CABG admission); long-term mortality (defined as all-cause mortality during follow-up duration); perioperative relevant events (such as renal impairment, permanent pacemaker implantation, stroke or transient ischemic attack, bleeding, sternal wound infection). S: Randomized controlled trial (RCT) or cohort study. Objective: The aim of this systematic review and meta-analysis is to evaluate the efficacy and safety of SA at the time of isolated CABG for preoperative AF patients based on the published literature, and provide reference for clinical decision.

Rationale: AF is the most common sustained cardiac arrhythmia. It can affect cardiac function, and is associated with higher risk of stroke and heart failure, which will immensely decrease patients' quality of life and survival. AF brings a heavy burden on health system, primarily due to its severe complications, such as stroke and sudden death. Treatments of AF include anti-arrhythmic drugs (AAD), catheter radiofrequency ablation, pulmonary vein isolation (PVI), Cox-Maze Procedure. AF frequently presents in association with other cardiovascular complications, such as coronary artery disease (CAD) and valvular disease. Preexisting AF has been found to be an independent risk factor for worse perioperative and long-term outcomes after cardiac surgery. However, whether adding a concomitant SA to the primary cardiac operations or not remains to be discussed. Although the major cardiology and surgery societies, including the Heart Rhythm Society (HRS), the Society of Thoracic Surgeons (STS), the American College of Cardiology (ACC), the American Heart Association (AHA) have recommended an ablation procedure during cardiac surgeries whenever feasible, the proportion of concomitant SA was still not very high, especially in the CABG procedure. The STS database from July 2011 to June 2014 indicated that only $48.3 \%$ patients with AF underwent surgical ablation during primary non-emergent cardiac operations. The results were as follows: mitral valve repair or replacement (MVRR) + CABG $68.4 \%$, aortic valve replacement (AVR) + CABG 39.3\%, MVRR + AVR $59.1 \%$, isolated CABG $32.8 \%$. Mitral operations had the highest rate of surgical ablation, while the proportion of SA at the time of isolated CABG was lower than it should be. Concern about additional incisions and complications, uncertain benefits and raised admission days and costs may be the primary reasons. Moreover, there is no uniform recommendation about selection of patients, incision set, ablation energy and strategy.

Condition being studied: There were multiple studies which enrolled patients scheduled for CABG combined with other cardiac procedure and reported different indexes. However, scant studies which systematically summarize benefits and harms specifically for dedicated CABG concomitant to AF ablation are available. Some reviews described the early and late outcomes of concomitant SA during CABG procedure, while they didn't compare the difference between CABG + SA group and CABG alone group. In addition, there are no consistent standards on the reporting of relevant outcomes. The detailed outcomes of surgical ablation in patients undergoing isolated CABG is less well established.

\section{METHODS}

Search strategy: ("atrial fibrillation" OR AF) AND ("coronary artery bypass" OR "coronary artery bypass grafting" OR "coronary artery bypass surgery" OR CABG) AND ("ablation techniques" OR "radiofrequency ablation" OR "maze procedure").

Participant or population: Patients with preoperative AF and indication for CABG.

Intervention: SA concomitant to CABG procedure.

Comparator: Isolated CABG procedure.

Study designs to be included: RCT or cohort study. 
Eligibility criteria: Inclusion criteria: (i) RCTs or cohort studies; (ii) patients with AF and indication for CABG; (iii) comparison of outcomes between isolated CABG + SA and isolated CABG alone in AF patients. Exclusion criteria: poor reporting of patients' characteristics, intraoperative and postoperative outcomes.

Information sources: We searched, with no language restrictions, the following database: the Cochrane Central Register of Controlled Trials (Central), PubMed, Embase, Web of Science, CBM, CNKI, VIP and WanFang database up to March 21, 2021. In addition, potentially eligible studies, such as conference literature and reference lists of included studies were reviewed manually.

Main outcome(s): Freedom from AF (defined as no AF recurrence during followup duration); early mortality (defined as any death within 30 days of CABG or during the CABG admission).

Additional outcome(s): Long-term mortality (defined as all-cause mortality during follow-up duration); perioperative relevant events (such as renal impairment, permanent pacemaker implantation, stroke or transient ischemic attack, bleeding, sternal wound infection).

Data management: We will sort and evaluate the retrieved articles using EndNote X8. Data will be extracted using Microsoft Excel 2019.

Quality assessment / Risk of bias analysis: We will use the Cochrane Collaboration's tool to assess methodological quality of RCTs and the Newcastle-Ottawa Scale (NOS) to assess methodological quality of cohort studies. The Cochrane Collaboration's tool assesses study quality from seven domains: random sequence generation, allocation concealment, blinding of participants and personnel, blinding of outcome assessors, incomplete outcome data, selective reporting and other bias. Each domain is categorized as low, high, or unclear risk of bias. The NOS contains eight items, categorized into three parameters including selection, comparability and outcome for cohort studies, which are assigned with a maximum of four, two, and three stars respectively. Therefore, nine stars reflects the highest quality. Studies with more than six stars will be considered of high quality.

Strategy of data synthesis: All data will be analyzed using the Review Manager version 5.3 (The Nordic Cochrane Centre, The Cochrane Collaboration, Copenhagen, Denmark). Analyses will be performed using risk ratios (RR) with a $95 \%$ confidence interval $(95 \%$ CI $)$ for dichotomous variables and mean difference (MD) with a 95\% Cl for continuous variables. All P-values are based on two-sided tests and $P<0.05$ is considered statistically significant. Heterogeneity will be evaluated by Cochrane $Q$ test and 12 test for each analysis. When $P \geq 0.1$ and $12 \leq 50 \%$, indicating minor heterogeneity between studies, we will use the fixed-effects model to calculate the pooled data. If $P<0.1$ or $I 2>$ $50 \%$, indicating moderate to higher heterogeneity between studies, the random-effects model will be used to calculate the data.

Subgroup analysis: We will perform subgroup analysis classified by AF types or ablation strategy if the relevant data is available.

Sensitivity analysis: If there is significant heterogeneity, we will perform sensitivity analysis to verity the stability of the combined effect.

Language: No language restriction was applied.

Country(ies) involved: China.

Keywords: Atrial fibrillation; Surgical ablation; Coronary artery bypass grafting; Meta-analysis.

Dissemination plans: The full article will be published in the public journal as a paper. 
Contributions of each author:

Author 1 - Kang Yi.

Author 2 - Xiao-Min Xu.

Author 3 - Jian-Guo Xu.

Author 4 - Shao-E He.

Author 5 - Wen-Xin Wang.

Author 6 - Yu-Hu Ma.

Author 7 - Jie Gao.

Author 8 - Wei Wang.

Author 9 - Tao You. 